\title{
Ruptured Conus Medullaris Dermoid Cyst with Fat Droplets in the Central
}

\author{
Mayur Sharma, Rahul Mally, Vernon Velho \\ Department of Neurosurgery, Sir J. J. Group of Hospitals, Grant Medical College, Mumbai, India
}

\begin{abstract}
Spinal dermoid tumors are rare, benign, slow growing tumors. These tumors may become acutely symptomatic after rupture or infection. Excision of the lesion with long term close follow-up studies is required for the management of these lesions. We present a very rare case of ruptured conus medullaris dermoid cyst in a 22-year-old male presented with urinary retention and low back pain. Magnetic resonance imaging scan with contrast reveals a lesion in the cauda equina inseparable from conus medullaris with fat droplets within the central spinal canal extending up to the medulla. Patient was operated with laminectomy and near complete excision of the lesion was done. Patient's low back pain was relieved following surgery. However patient had persistent urinary incontinence and on clean intermittent self-catheterization. Histopathology was suggestive of dermoid cyst.
\end{abstract}

Keywords: Ruptured; Spinal cord; Dermoid cyst

\section{Introduction}

Dermoid tumors constitute $1.1 \%$ of intraspinal tumors [1]. They arise from the inclusion of ectopic embryonic rests of ectoderm within the spinal canal at the time of neural tube closure during embryonic development [2]. The lumbosacral region is the most common site to be affected $(60 \%)$ [3].

They are benign slow growing lesions which become clinically apparent during the second or third decade of life [4] and males are more commonly affected.

We present a very rare case of ruptured conus medullaris dermoid cyst in a 22-year-old male presenting with urinary retention. Radiological examination was suggestive of a ruptured dermoid cyst with fat droplets in the central spinal canal extending up to the medulla.

\section{Case Report}

A 22-year-old male patient was admitted with history of sudden onset urinary retention 7 months back followed by urinary catheterization for the same. Patient was weaned off the urinary catheter in 7 days, following which patient developed urinary incontinence for 6 months. It was not associated with urgency, frequency or burning micturition.

Patient also noticed low backache for last 7 days before coming to us. Pain was mild, intermittent aching in nature, not radiating to lower limbs and used to relieve with analgesics. No aggravating or relieving factors for low backache.

On neurological examination, higher mental functions and cranial nerves were normal. Power was 5/5 at all the joints in all the four limbs. Sensory examination did not reveal any abnormality. Both superficial and deep tendon

Received Sep 25, 2011; Revision Nov 26, 2011; Accepted Jan 26, 2012

Corresponding author: Mayur Sharma

Department of Neurosurgery, Sir J. J. Group of Hospitals, Grant Medical College,

Byculla, Mumbai 400008, India

Tel: +91-22-23735555, Fax: +91-22-23735599, E-mail: drmayur_sharma@yahoo.co.in, drmayursharmaneuro@gmail.com 
reflexes were normal. Gait was normal. Straight leg raising test was free on both sides.

\section{X-ray lumbo-sacral spine was normal}

Magnetic resonance imaging (MRI) scan (Figs.1-4) with

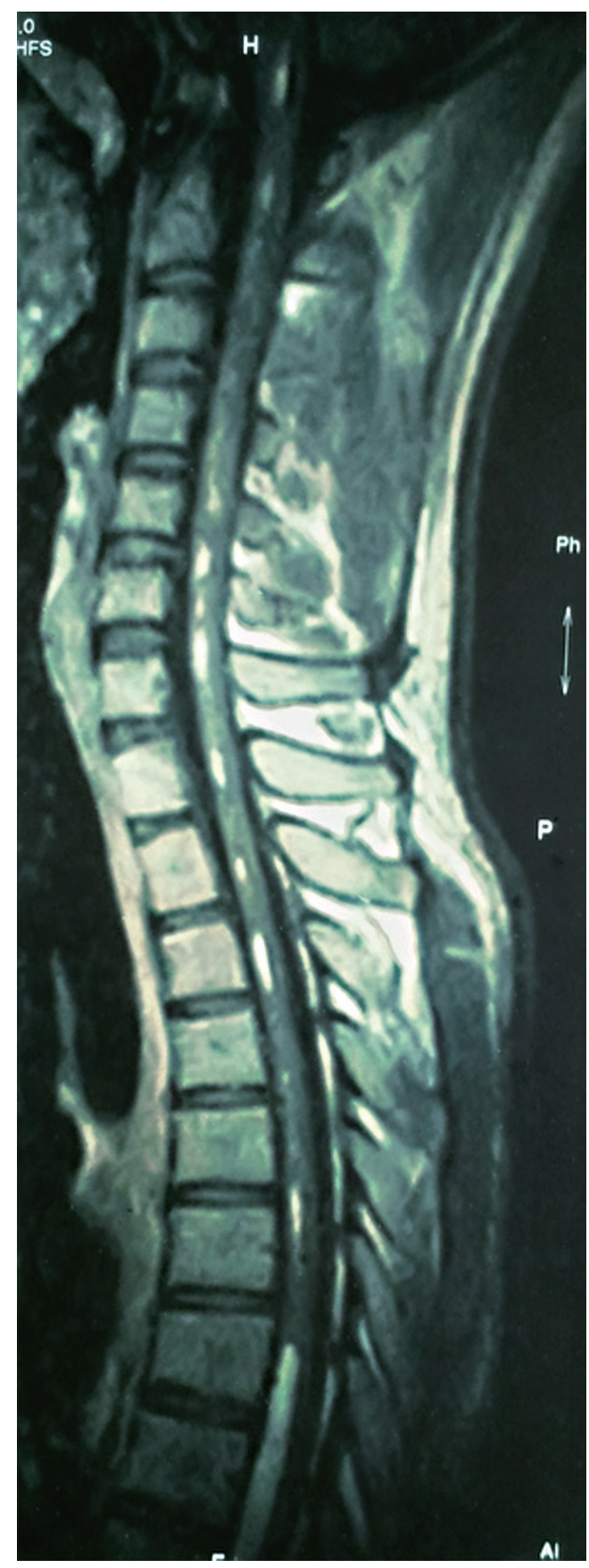

Fig. 1. Magnetic resonance imaging. T1 weighted (sagittal view) images showing hyperintense fat content in the central canal extending up to the medulla secondary to rupture of the mass. contrast and fat suppression studies was showing a well circumscribed lesion approximately $5.4 \times 2.1 \times 1.8 \mathrm{~cm}$ in the cauda equina inseparable from conus medullaris with fat droplets within the central spinal canal extending up to the medulla. The lesion was iso to hypo intense on T1 and hyperintense on T2 weighted images. There was no significant post contrast enhancement. T1 hyper intense signal is seen scattered throughout the central spinal canal extending upto the medulla. MRI scan on T1 weighted (sagittal view) images showed a mass lesion containing fat peripherally, at the level of conus medullaris and hyperintense fat content in the central canal extending in to the dorsal and cervical spinal cord up to the medulla secondary to rupture of the mass (Fig. 1).

\section{Provisional preoperative diagnosis was ruptured in- traspinal dermoid}

Patient was operated with D11-L2 laminectomy and near total excision of the lesion. The spinal cord was expanded and myelotomy was done at the conus medullaris. Intraoperatively, the lesion was intramedullary, white cheesy with hair inside. The capsule was densely adherent to the spinal cord, therefore near complete excision was achieved.

\section{Lesion was reported as dermoid cyst on frozen sections}

Histopathological examination showed a fibro collagenous cyst wall lined by stratified squamous epithelium.

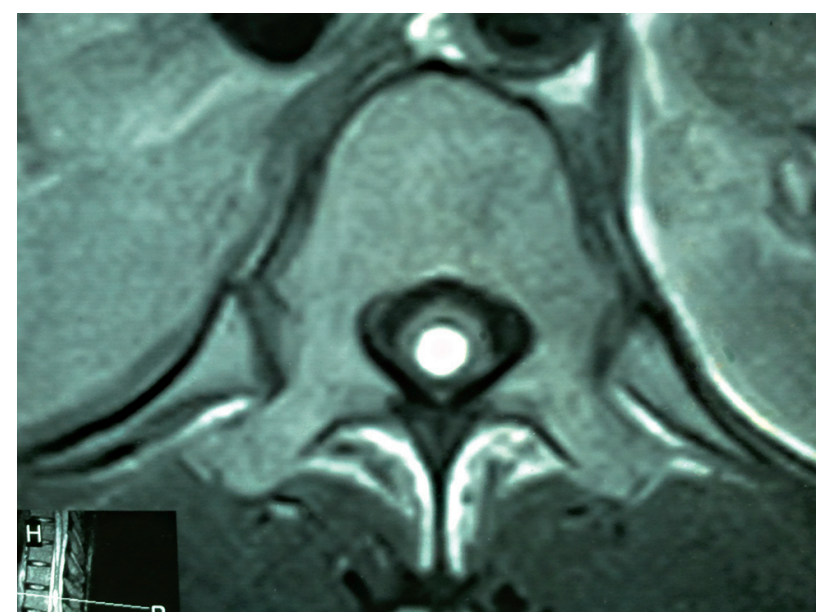

Fig. 2. Magnetic resonance imaging scan T1 weighted (axial view) images at D-12 vertebral level showing hyper intense fat content in the central canal. 
in view of the presence of hair on gross examination the diagnosis of dermoid cyst was given (Fig. 5).

Patient's low back pain had improved immediately following the surgery. There was no neurological deficit in the lower limbs after the surgery. However patient had persistent urinary incontinence and on clean intermittent self catheterisation at the time of discharge.

At 6 month follow-up, patient was relieved of his low

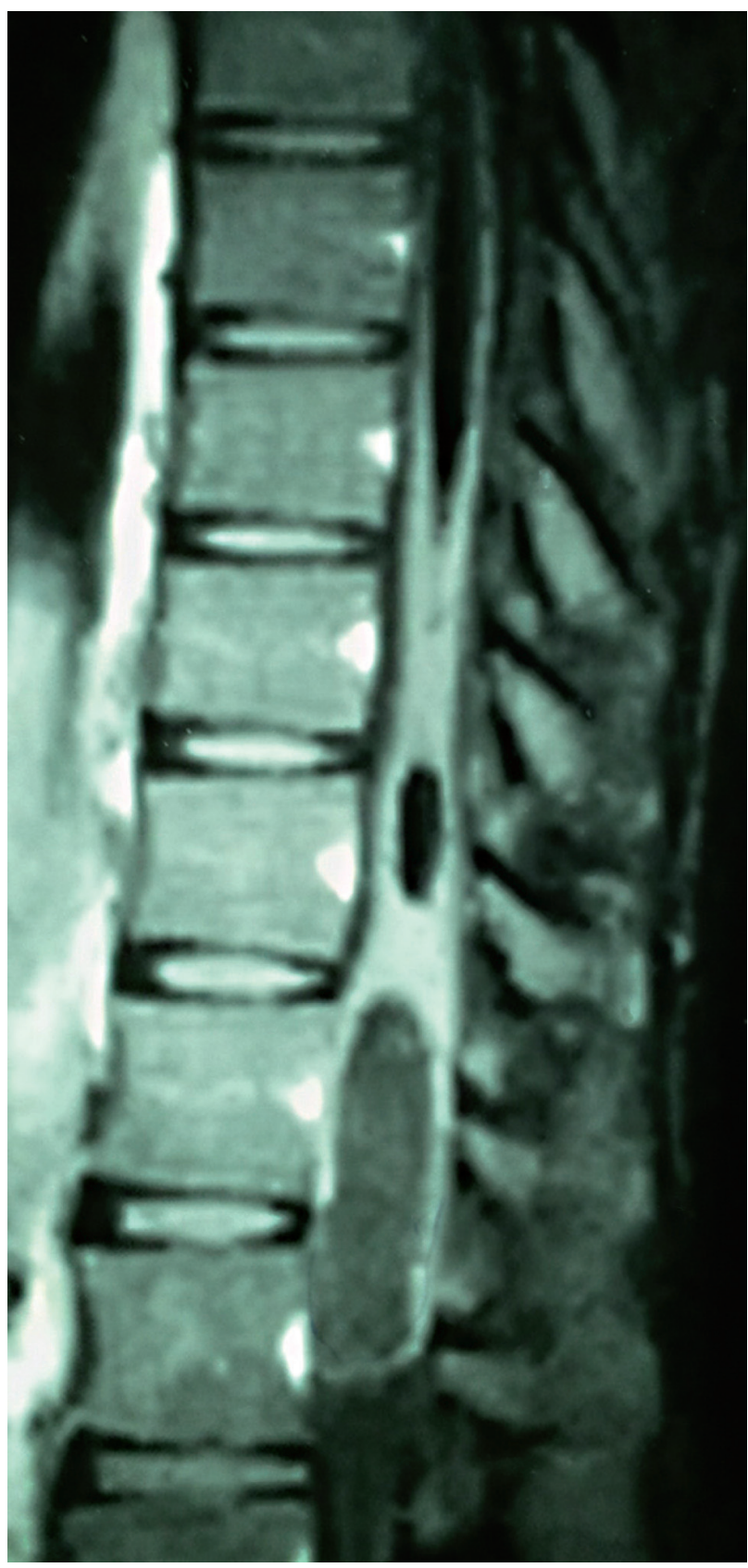

Fig. 3. Magnetic resonance imaging scan T1-weighted fat suppressed (sagittal view) images at conus medullaris extending in to the dorsal spinal cord demonstrates signal suppression of the fat content and no significant enhancement. back pain with improvement in the bladder functions and weaned off the urinary catheter.

\section{Discussion}

Dermoid tumors constitute $1.1 \%$ of intraspinal tumors [1]. They arise from the inclusion of ectopic embryonic rests of ectoderm within the spinal canal at the time of neural tube closure during embryonic development [2].

They occur predominantly in the lumbosacral region (60\%), involving the cauda equina and conus medullaris [3], and are quite rare in the upper thoracic (10\%) and cervical regions $(5 \%)[3,4]$. As in our case patient had dermoid cyst in the conus medullaris.

They are benign slow growing lesions which become clinically apparent during the second or third decade of

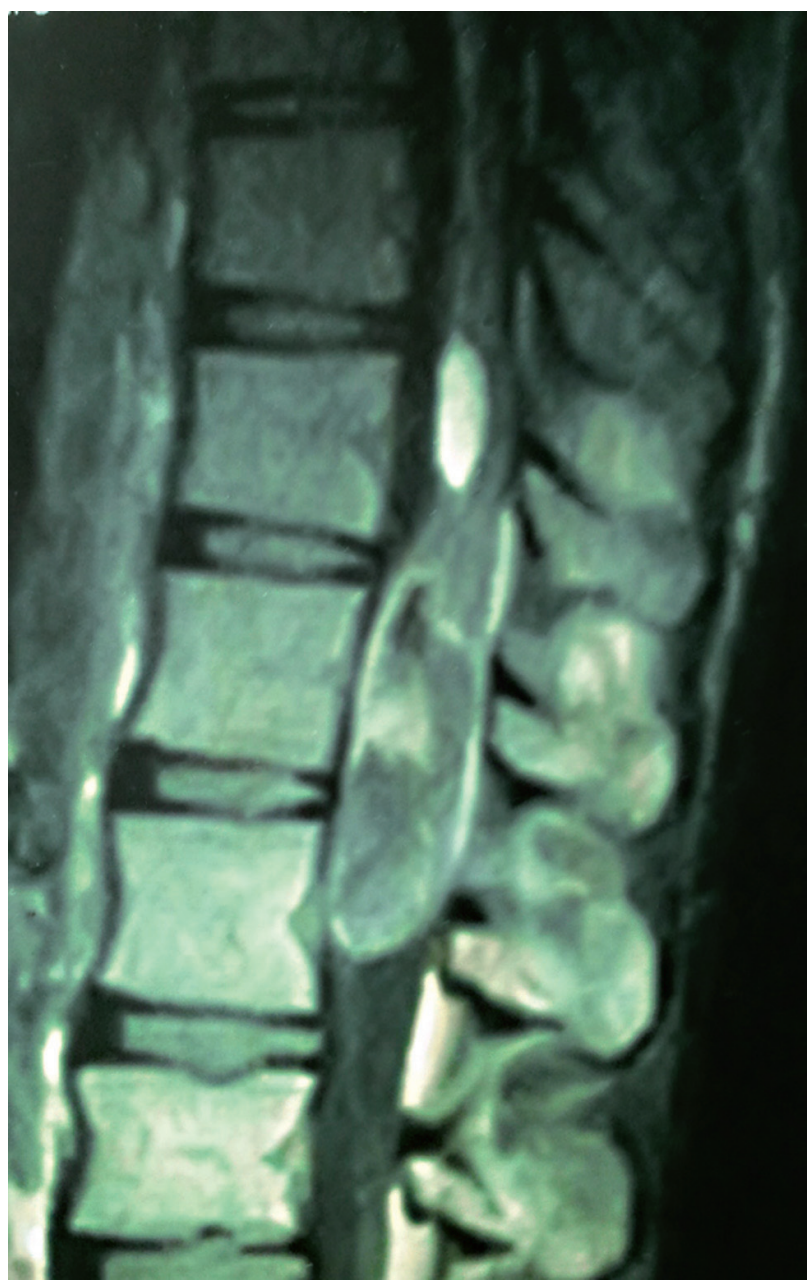

Fig. 4. Magnetic resonance imaging scan T1-weighted post-contrast (sagittal view) images at conus medullaris showing no significant post contrast enhancement. 
life [5]. And males are more commonly affected, as in our case patient was 22-year-old male.

These may be associated with a dorsal dermal sinus in about $20 \%$ of the cases [6] along with bony malformations and tethered cord [7].

The clinical history of our patient was related to the localization of the lesion.

Dermoid tumors can reach rather large sizes without producing any symptoms or findings.

Although their nature is benign and development is slow, dermoid tumors have a high morbidity and mortality risk, especially when rupture occurs. A dermoid cyst can rupture during surgery, after a trauma or spontaneously [8-10].

Clinical symptoms of acute rupture are headache, nausea, vomiting, vertigo, vision problems, aseptic chemical meningitis, hemiplegia, mental changes, and coma $[8,9]$. Later on, the existence of fat droplets in the subarachnoidal and ventricular spaces may lead to arachnoiditis, ventriculitis, and, consequently, mental disorder may occur.

MRI is the investigation of choice for detecting intraspinal dermoid tumors and delineating their extent [10]. Computed tomography (CT) scan is very useful in identifying areas of fat and calcification. The presence of fat, calcification, soft tissue and fluid gives a clue to the diag-

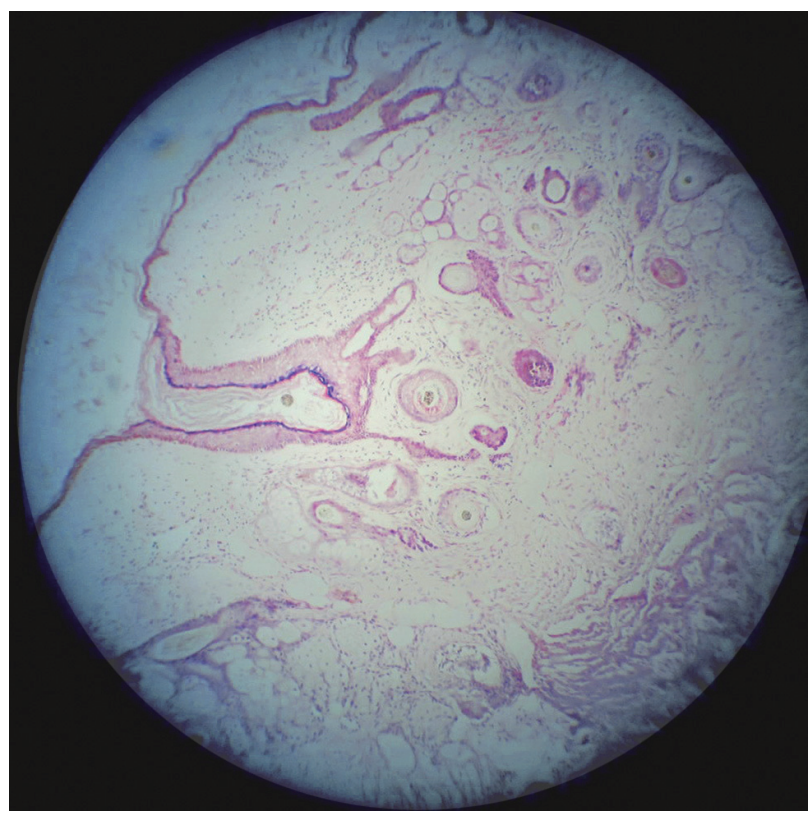

Fig. 5. Histopathological examination showing a fibro collagenous cyst wall lined by stratified squamous epithelium with hair follicles and sebaceous glands in the dermis suggestive of dermoid cyst ( $\times 10)$. nosis of dermoid cyst.

MRI typically reveals heterogeneous signal intensity because of the presence of fat, calcium and fluidv [6]. High lipid content emanated from sebaceous glands causes high signal intensity on T1W spin echo images. Bone and cartilage might be found in the tumor itself and sometimes calcification can be seen in their walls. Dermoid tumors can be made up of 2 different pieces, one with a higher lipid content and the other with a solid or more liquid content, and this condition may lead to a liquid-liquid level [3].

In our case, on fat-suppressed sequences, fat content of the tumor was clearly demonstrated. The relatively high signal intensity from fat, with the bright signal on T1W images and fat suppression on fat suppression studies makes identification of the lipid component easy [7], as in our case.

Diagnostic accuracy of MR imaging will increase with the use of IV contrast medium, especially in determining meningeal inflammatory reaction related to the spreading out of tumoral contents

The differential diagnosis of spinal dermoid cysts includes lipomas, teratomas, and spinal cord tumors (astrocytoma, ependymoma).

The reported number of dermoid tumors is increasing due to the frequent use of MR imaging. Free fatty material within the ventricular system and intracranial cisterns were reported in all cases, but fat particles in the dilated central spinal canal were reported in only few studies [8].

With the frequent use of MR imaging it was found that spontaneous dermoid rupture, which was previously thought of as a rather serious or fatal condition, is common and usually only slightly symptomatic or asymptomatic.

Histopathological findings are very classic of dermoid cyst with fibro collagenous cyst wall lined by stratified squamous epithelium with the presence of hair on gross examination as in our case.

Patients presenting with features of spinal cord compression or radiculopathy or neurological deficit require surgical intervention for tissue diagnosis and decompression. In our case, we have done D11-L2 laminectomy and near total excision of the lesion with neurological improvement.

However, because of the paucity of the number of cases of the ruptured dermoid cyst in the central canal, the management of ruptured fat droplets into the central ca- 
nal is still not definite.

The management consists of excision of the dermoid cyst as completely as possible. However if the wall of the lesion is densely adherent to cord, near total excision in such cases is acceptable $[1,4]$.

Long term follow-up is also not available because of few numbers of cases.

Ruptured dermoid cyst in the central canal is a very rare entity, however it should be kept in the differential diagnosis if the patient becomes acutely symptomatic with headache, nausea, vomiting, either following trauma or spontaneously. MR imaging is the most important radiologic modality to diagnose, to determine the distribution of ruptured tumor content into the subarachnoid space, or into the central spinal canal, to determine complications such as hydrocephaly or meningitis after rupture, and for follow-up after surgery.

Surgical excision of the lesion for the diagnosis with close observation and follow up studies to detect complications should result in significant neurological improvement

\section{Conflict of interest}

No potential conflict of interest relevant to this article was reported.

\section{Acknowledgments}

We would like to thank our Dean, Sir J.J. group of hospitals for allowing us to publish the hospital record.

\section{References}

1. Lunardi P, Missori P, Gagliardi FM, Fortuna A. Long-term results of the surgical treatment of spinal dermoid and epidermoid tumors. Neurosurgery 1989;25:860-4.

2. Netsky MG. Epidermoid tumors. Review of the literature. Surg Neurol 1988;29:477-83.

3. Graham DV, Tampieri D, Villemure JG. Intramedullary dermoid tumor diagnosed with the assistance of magnetic resonance imaging. Neurosurgery 1988;23:765-7.

4. Kumar S, Gulati DR, Mann KS. Intraspinal dermoids. Neurochirurgia (Stuttg) 1977;20:105-8.

5. Egelhoff JC. Pediatric head and neck imaging. In: Haaga JR, editor. CT and MR imaging of the whole body. 4th ed. London: Mosby; 2003. p.696.

6. McAdams HP. Mediastinum. In: Haaga JR, editor. CT and MR imaging of the whole body. 4th ed. London: Mosby; 2003. p.959-60.

7. Cha JG, Paik SH, Park JS, Park SJ, Kim DH, Lee HK. Ruptured spinal dermoid cyst with disseminated intracranial fat droplets. Br J Radiol 2006;79:167-9.

8. Scearce TA, Shaw CM, Bronstein AD, Swanson PD. Intraventricular fat from a ruptured sacral dermoid cyst: clinical, radiographic, and pathological correlation. Case report. J Neurosurg 1993;78:666-8.

9. Barsi P, Kenez J, Varallyay G, Gergely L. Unusual origin of free subarachnoid fat drops: a ruptured spinal dermoid tumour. Neuroradiology 1992;34:343-4.

10. Wilms G, Casselman J, Demaerel P, Plets C, De Haene I, Baert AL. CT and MRI of ruptured intracranial dermoids. Neuroradiology 1991;33:149-51. 